\title{
A Case Study of Error Analysis and Error Correction in College Students' Argumentative Writing
}

\author{
Xiaoyuan Deng \\ North University of China, Taiyuan, China \\ Email: 1712886355@qq.com
}

How to cite this paper: Deng, X.Y. (2021) A Case Study of Error Analysis and Error Correction in College Students' Argumentative Writing. Open Access Library Journal, 8: e7936.

https://doi.org/10.4236/oalib.1107936

Received: September 7, 2021

Accepted: September 15, 2021

Published: September 18, 2021

Copyright $\odot 2021$ by author(s) and Open Access Library Inc.

This work is licensed under the Creative Commons Attribution International License (CC BY 4.0).

http://creativecommons.org/licenses/by/4.0/

\section{(c) (i) Open Access}

\begin{abstract}
English writing is an important means to measure the English learning level of learners. Among the five basic language skills of "listening, speaking, reading, writing and translation", "writing" is the most important language output in language learning, which can reflect the learners' comprehensive application ability of language. However, learners often make mistakes in their English compositions, which can largely reflect their level of writing. Based on the theory of error analysis, this study conducts error analysis through eight composition samples of the research subjects, finds out the characteristics of error distribution, explores the causes of error, finds methods and strategies to improve the writing of English learners, and further promotes the teaching of English writing.
\end{abstract}

\section{Subject Areas}

Linguistics

\section{Keywords}

English Writing, Error Analysis, Error Correction

\section{1. 引言}

\section{1. 英语写作}

从语言教育的角度来看, 读写能力在母语教育中是 “文化” 的象征, 尤 其是写作能力 $[1]$ 。在 “二语教与学” 中, 写作作为一个重要环节, 是学习者 语言能力的直接体现, 但是对多数同学来说, 写作是个难题[2]。Ulign \& Strother [3] 认为写作通常是一种积极有效的语言表达技能。它要求学习者不 
仅要充分掌握一定数量的词汇, 而且要有语法思维能力、认知思维能力以及 语言表达、逻辑推理及分析能力。英语写作的定义, 可从广义和狭义两方面 划分。从广义上讲, 写作是作者按照本身的主观意识, 经由恰当的语言表达, 对客观事物的反应。从狭义上讲, 英语写作是为达到课程标准的要求, 语言 学习者进行的一项练习活动。写作是一项重要的语言技能, 是一种没有声音 的表达方式, 其本身就是一次复杂的过程, 需要思维的运转, 创造力与实践 力的结合, 将想法整理, 再转换成信息。因此对英语学习者来说, 英语写作 是一次相对不易的输出过程。英语写作通常情况下分别为说明文、描述文、 记叙文和议论文等四种。而本文收集的作文全是议论文。

\section{2. 错误分析理论}

错误分析理论(Error Analysis)作为一种外语学习方法, 产生于二十世纪六 十年代 [4] [5]。基于认知心理学和生成语言学, 错误分析理论提出人类的大脑 中具有能够处理语言功能的特殊机制 [6], 其通过评估分析学习者在二语习得 中的错误, 找出其中的规律, 发现他们在语言学习过程中使用的策略并分析 产生错误的原因, 并在理论上做出一些陈述和解释, 以指导教学实践。作为 错误分析理论的最早提出者, Pit Corder 认为, 错误, 即偏离目的语的语言表 达, 是学习者创造性使用语言的体现, 其存在于语言学习的每一个阶段, 是 不可避免的, 也是学习过程中非常重要的一部分, 而这种错误能给教学者及 学习者提供大量有用的信息。Candling [7]认为学习者的偏误对于二语习得进 程的理解具备潜在的重要性, 同时他也提出学习者犯错是学习曲线中的不可 或缺的部分。根据 James [8], 错误分析便是人们对语言无知的深究, 考察人 们是怎样试图解决语言无知的。错误分析的步骤可分为搜集资料、错误辨别、 错误分类、错误归因和错误纠正等五步。Corder (2004) 以为, 错误分析理论 的钻研, 对语言学习和语言教学都必不可少, 同时对其发展有着非常大的积 极作用。首先, 教师对英语学习者的错误进行系统的分析, 所得出的结果能 够反应出学习者在目标语的学习中处于哪个阶段, 从而掌握其目标语的学习 进度及情况; 其次, 英语学习者的错误分析结果能够体现其所运用的学习方 法与策略, 以及在写作过程中的心理状态, 从而提供给教师可靠的反馈, 能 针对不同的错误类型设计修订教学方法。其次, 对于英语学习者来说, 错误 分析能够帮助其了解自身在英语写作中常犯的错误类型, 发现错误产生的原 因, 从而检验学习者学习水平。因而, 错误分析对于外语学习者和教师来说, 都是应该掌握的一项分析和评价工具。关于处理学习者的错误, 很多教师认 为应注意纠正学生写作中全部呈现出来的错误。一些学者 [9] [10]则提出, 教 师应该为了提高学习者学习语言的兴趣, 逐步建立其学习外语的自信, 而尽 量容忍某些小错误。此外, 一些教育者验证了各类错误纠正的方式, 其中包 含直接纠错、错误反馈和不反馈, 最终发现错误反馈和自我纠错相对于直接 纠错和不纠正而言, 更有助于学习者的英语写作。因而, 笔者在辅导时采用 了自我纠错、集体讨论和直接纠错这三种方式来对参与者的英语写作进行帮 助和提升。 


\section{2. 研究方法}

\section{1. 研究问题}

1) 英语专业学习者在英语写作中的主要错误是什么?

2) 经过课外辅导及英语写作错误修正, 学生的英语写作会得到帮助吗?

\section{2. 研究设计}

本研究参与者是来自中北大学英语专业的同一班级的两位同学 Lauren 和 Anna。笔者通过对两位参与者进行英语写作辅导, 收集了参与者经过多次修 订的文章和录音。数据收集历时 3 周。首先, 两位参与者根据既定话题写一 篇 300 字左右的文章, 话题围绕 “The Most Important Personal Quality of a University Study”。随后笔者对参与者进行了第一次辅导, 主要围绕如何有 逻辑性、有条理性地开展 0 作文写作, 并让参与者进行了自我纠正, 找出错 误原因, 进行对文章的第一次改写。再次, 在第二次辅导中, 研究对象先后 采取了集体纠错和自我纠错, 笔者仍然采用相同的方式解决其作文中出现的 错误。研究对象进行第二次改写。在最后一次写作辅导中, 笔者和研究对象 共同纠错, 发现问题, 讨论了作文的第三次改写。在交流与讨论之后, 研究 对象对作文进行了第四次改写, 由此, 就形成了作文的最终版本。

\section{3. 数据分析}

本实验最后共收集到两位参与者同一主题的八篇作文样本作为研究数 据。笔者统计并分析了这些文章所存在的错误, 一般分为三类: 词汇方面的 错误(包含动词、副词、名词、形容词、短语), 形式方面的错误(包括介词、 冠词、名词和动词形式) 以及语篇方面错误(包括时态、语篇结构、语篇逻辑)。

\section{3. 研究结果}

研究结果表明, 总体上, Lauren 和 Anna 错误总计 56 处, 其中 Lauren 所占 $68 \%$ (见表 1)。在两位参与者的文章中所出现的错误中, 词汇错误最少。 Lauren 文章中所犯的形式错误最多。Anna 的语篇问题占到 $61 \%$, 所以在英语 写作时形式错误和语篇构建问题也要引起重视。

\section{1. 词汇错误}

参与者写作中的词汇错误主要存在于两方面: 用词不当和词语的搭配错

表 1. 两位参与者文章中的形式错误和语篇问题统计

\begin{tabular}{ccccccccc}
\hline & \multicolumn{4}{c}{ Lauren } & \multicolumn{3}{c}{ Anna } \\
\cline { 2 - 8 } & 词汇 & 形式 & 语篇 & 总数 & 词汇 & 形式 & 语篇 & 总数 \\
\hline 第二稿 & 4 & 8 & 2 & 14 & 1 & 1 & 3 & 5 \\
第三稿 & 3 & 7 & 1 & 11 & 0 & 1 & 2 & 3 \\
第四稿 & 0 & 2 & 1 & 3 & 0 & 0 & 2 & 2 \\
总数 & 8 & 21 & 9 & 38 & 3 & 4 & 11 & 18 \\
\hline
\end{tabular}


误, 同时冠词的用法也存在错误。具体实例如下:

(1) Everyone should make the best use of time to learn knowledge.

(2) I'm looking forward to find some strategies and ways to get rid of the bad habit.

(3) And I believe he can make a success.

句(1)中 “learn knowledge” 为错误的动词和名词搭配, 更为恰当的表达 方式为 “acquire knowledge”。句(2)中的 “look forward to” 是英语中常见的 固定搭配, “to” 是介词, 后面应跟 “finding some strategies”。句(3)中应该 用固定搭配 “gain/achieve success”。

\section{2. 形式错误}

语法错误包括词语的形态错误(主要包括名词、动词、形容词和副词这四 类词的形态变化错误，以及名词单复数、动词第三人称单数等形态错误)及句 法错误(语态错误、语序错误和句子结构错误等)。典型错误例句如下：

(1) Nobody like those people who are often complaining about the unfairness of life.

(2) Difficulties are inevitable on the way to success. Everyone needs to be determined no matter what hard ships you have encountered.

(3) He has some evidences to point his view.

句(1)中出现了主谓不一致的错误。不定人称代词 nobody 作为主语的时 候，其后的谓语动词应该使用第三人称单数形式 “likes”。句(2)应当使用一 般将来时的时态即 “will encounter” , 这是时态错误。句(3)出现了单复数形 式错误和词汇搭配不当, 正确形式应该是 “I have some evidence to support my view”。

\section{3. 语篇错误}

篇章是学生对写作内容整体性的把握, 语篇错误主要与语句的衔接、连 贯和语用有关。在研究对象的文章中, “First, some students had no plan, they were a little anxious.” 前半句"同学们没有计划"与后半句 “感到焦虑” 存在明 显的因果关系，故这句话缺少表现因果关系的连接词。英语重形合，汉语重 意合, 因而, 英语句子通常表现出严谨而理性的特点, 而汉语则显得更为感 性、结构松散。两位参与者也因为母语迁移的因素而使文章缺乏连接词显得 松散。在四次纠错前, 两位参与者的文章结构较为松散, 前后内容缺乏连贯 性，段落与段落之间没有过渡句，句与句之间没有恰当的连接词或者连接短 语。而在四次写作纠错后，收集到的作文结构基本完整，布局方面基本没有 问题, 只存在少量语病。

\section{4. 错误纠正}

从表 2 可知, 在收到的八篇作文中, 自我纠错相较于其他两种方式, 显 得更有成效，集体讨论次之，而居于末位的是直接指出参与者作文中错误的 方式。由表 2 还可以表明自我纠错的指出的错误所占总数的比例最低, 由此 
表 2. 对两位参与者文章纠错的数据统计

\begin{tabular}{cccccc}
\hline & \multicolumn{2}{c}{ Lauren } & \multicolumn{3}{c}{ Crystal } \\
\cline { 2 - 6 } & 指出数目 & 纠正数目 & 指出数目 & 纠正数目 & 纠错率 \\
\hline 自我纠错 & 4 & 4 & 5 & 5 & $100 \%$ \\
集体讨论 & 15 & 13 & 3 & 2 & $83 \%$ \\
直接纠错 & 18 & 13 & 9 & 4 & $63 \%$ \\
\hline
\end{tabular}

说明自我纠错对于语言学习者来说较为困难, 这是因为纠错时学生自身存在 的的一贯思维, 较难发现自己作文中的错误, 这也反映出学生在词汇和语法 等方面的基础知识相对薄弱。

\section{4. 结语}

至此, 本文已可以回答本文两个研究问题。第一, 在本次个案研究中, 参与对象在英语写作上的难点在于句子及语篇结构。第二, 最终的文章虽还 存在一些不足, 但对于本次研究对象来说, 通过辅导, 四次的文章内容中的 错误逐步减少, 由此可见, 本次辅导对他们而言有较大的帮助。此次课外辅 导也让研究对象意识到了在英语写作中, 思维逻辑和语篇结构是非常关键的。 英语学习是一个漫长的内化过程, 在此期间, 学习者会朝着掌握目标语的完 整体系而努力, 而错误出现的情况很常见[11], 但语言能力正是在错误的纠正 中进而不断提高。同时, 本次研究对于外语写作的教与学有着重要意义。对 于教师来说, 在外语写作教学中, 运用错误分析理论能够帮助教师更有效地 组织教学, 更高效地将学习者写作的错误归类, 分析其产生的原因, 采取不 同的处理方式。对学习者来说, 错误分析也是自身语言学习的一种激励机制。 除此之外, 教师和学生都应该认识到, 对待语言习得中出现的问题应抱有理 智宽容的态度, 恰当地将错误理论应用到教学中, 才能逐步解决学生学习外 语中出现的错误。

\section{Conflicts of Interest}

The author declares no conflicts of interest.

\section{References}

[1] 张德禄. 多模态学习能力培养模式探索 [J]. 外语研究, 2012(2): 9 .

[2] 王文宇, 俞希. 大学生二语写作难点的一项调查 [J]. 外语教学理论与实践, 2008(1): 31-35.

[3] Ulijin, J. and Strother, J.M. (1995) Communication in Business and Technology. Lang, Frankfurt.

[4] Corder, S.P.M. (1973) Introducing Applied Linguistics. Penguin Books, London.

[5] Corder, S.P.J. (1967) The Significance of Learner's Errors. International Review of Applied Linguistics, No. 4, 161-170. https://doi.org/10.1515/iral.1967.5.1-4.161

[6] 戴炜栋, 束定芳. 对比分析、错误分析和中介语研究中的若干问题一一外语教学 理论研究之二[J]. 外国语, 1994(5): 1-7. 
[7] Candling, R.M. (2001) Vocabulary and Language Teaching. Longman Inc., New York.

[8] James, C.M. (2001) Errors in Language Use: Exploring Error Analysis. Addison Weslsey Longman Limited, Harlow, Essen.

[9] Krashen, S.D.M. (1987) Principles and Practice in Second Language Acquisition. Prentice Hall, Great Britain.

[10] Truscott, J. (1996) The Case against Grammar Correction in L2 Writing Classes. Language Learning, No. 46, 327-369.

https://doi.org/10.1111/j.1467-1770.1996.tb01238.x

[11] 桂诗春. 应用语言学与中国外语教学[M]. 济南: 山东教育出版社, 1988.

\section{Appendix (Abstract and Keywords in Chinese) 大学生英语议论文写作错误分析和错误纠正之个案研究}

摘要: 英语写作是衡量学习者英语学习水平的一项重要手段, 在 “听、说、 读、写、译” 五大基本语言技能中, “写” 是语言学习中最重要的语言输出, 能反应出学习者的语言综合运用能力。然而学习者的英语作文中会时常出现 各种错误, 这些错误能很大程度上反映出其写作水平。本研究以错误分析的 理论为基础, 通过研究对象的八篇作文样本进行错误分析, 找出错误分布特 点, 探究错误成因, 寻找提高英语学习者写作的方法与策略, 进一步促进英 语写作教学。

关键词: 英语写作, 错误分析, 错误纠正 